EPJ Web of Conferences 71, 00050 (2014)

DOI: 10.1051/epjconf/20147100050

(C) Owned by the authors, published by EDP Sciences, 2014

\title{
Predictions for diffraction at the LHC compared to experimental results
}

\author{
Konstantin Goulianos ${ }^{1, a}$ \\ ${ }^{1}$ The Rockefeller University, 1230 York Avenue, New York, NY 10065, USA
}

\begin{abstract}
Diffractive proton-proton cross sections at the LHC, as well as the total and total-inelastic proton-proton cross sections, are predicted in a simple model obeying all unitarity constraints. The model has been implemented in the PYTHIA8-MBR event generator for single diffraction, double diffraction, and central diffraction processes. Predictions of the model are compared to recent LHC results.
\end{abstract}

\section{Introduction}

Measurements at the LHC have shown that there are sizable disagreements among Monte Carlo (MC) implementations of "soft" processes based on cross sections proposed by various physics models, and that it is not possible to reliably predict all such processes, or even all aspects of a given process, using a single model [1-3]. In the CDF studies of diffraction at the Tevatron, all processes are well modeled by the MBR (Minimum Bias Rockefeller) MC simulation, which is a stand-alone simulation based on a unitarized Regge-theory model, RENORM [4], employing inclusive nucleon parton distribution functions (PDF's) and QCD color factors. The RENORM model was updated in a presentation at EDs-2009 [5] to include a unique unitarization prescription for predicting the total $p p$ cross section at high energies, and that update has been included as an MBR option for simulating diffractive processes in PYTHIA8 since version PYTHIA8.165 [6], to be referred here-forth as PYTHIA8-MBR. In this paper, we briefly review the cross sections [7] implemented in this option of PYTHIA8 and compare them with LHC measurements.

The PYTHIA8-MBR option includes a full simulation of the hadronization of the implemented diffraction dissociation processes: single, double, and central diffraction. In the original MBR simulation used in $\mathrm{CDF}$, the hadronization of the final state(s) was based on a data-driven phenomenological model of multiplicities and $p_{t}$ distributions calibrated using $\mathrm{S} \bar{p} p \mathrm{~S}$ and Fermilab fixed-target results. Later, the model was successfully tested against Tevatron Mв and diffraction data. However, only $\pi^{ \pm}$and $\pi^{0}$ particles were produced in the final state, with multiplicities obeying a statistical model of a modified Gamma distribution function that provided good fits to experimental data [8]. This model could not be used to predict specific-particle final states. In the PYTHIA8-MBR implementation, hadronization is performed by PYTHIA8 tuned to reproduce final-state distributions in agreement with MBR's, with hadronization done in the PYTHIA8 framework. Thus, all final-state particles are now automatically produced, greatly enhancing the horizon of applicability of tPYTHIA8-MBR.

\footnotetext{
a e-mail: dino@rockefeller.edu
} 


\section{Cross sections}

The following diffraction dissociation processes are considered in PYTHIA8-MBR:

$$
\begin{array}{rll}
\text { SD } & p p \rightarrow X p & \text { Single Diffraction (or Single Dissociation), } \\
\text { or } & p p \rightarrow p Y & \text { (the other proton survives) } \\
\text { DD } & p p \rightarrow X Y & \text { Double Diffraction (or Double Dissociation), } \\
\text { CD (or DPE) } & p p \rightarrow p X p & \text { Central Diffraction (or Double Pomeron Exchange). }
\end{array}
$$

The RENORM predictions are expressed as unitarized Regge-theory formulas, in which the unitarization is achieved by a renormalization scheme where the Pomeron $(\boldsymbol{P})$ flux is interpreted as the probability for forming a diffractive (non-exponentially suppressed) rapidity gap and thereby its integral over all phase space saturates at the energy where it reaches unity. Differential cross sections are expressed in terms of the $\boldsymbol{P}$-trajectory, $\alpha(t)=1+\epsilon+\alpha^{\prime} t=1.104+0.25\left(\mathrm{GeV}^{-2}\right) \cdot t$, the $\boldsymbol{P}$ - $p$ coupling, $\beta(t)$, and the ratio of the triple- $\boldsymbol{P}$ to the $\boldsymbol{P}$ - $p$ couplings, $\kappa \equiv g(t) / \beta(0)$. For large rapidity gaps, $\Delta y \geq 3$, for which $\boldsymbol{P}$-exchange dominates, the cross sections may be written as,

$$
\begin{aligned}
\frac{d^{2} \sigma_{S D}}{d t d \Delta y} & =\frac{1}{N_{\text {gap }}(s)}\left[\frac{\beta^{2}(t)}{16 \pi} e^{2[\alpha(t)-1] \Delta y}\right] \cdot\left\{\kappa \beta^{2}(0)\left(\frac{s^{\prime}}{s_{0}}\right)^{\epsilon}\right\}, \\
\frac{d^{3} \sigma_{D D}}{d t d \Delta y d y_{0}} & =\frac{1}{N_{\text {gap }}(s)}\left[\frac{\kappa \beta^{2}(0)}{16 \pi} e^{2[\alpha(t)-1] \Delta y}\right] \cdot\left\{\kappa \beta^{2}(0)\left(\frac{s^{\prime}}{s_{0}}\right)^{\epsilon}\right\}, \\
\frac{d^{4} \sigma_{D P E}}{d t_{1} d t_{2} d \Delta y d y_{c}} & =\frac{1}{N_{\text {gap }}(s)}\left[\Pi_{i}\left[\frac{\beta^{2}\left(t_{i}\right)}{16 \pi} e^{2\left[\alpha\left(t_{i}\right)-1\right] \Delta y_{i}}\right]\right] \cdot \kappa\left\{\kappa \beta^{2}(0)\left(\frac{s^{\prime}}{s_{0}}\right)^{\epsilon}\right\},
\end{aligned}
$$

where $t$ is the 4-momentum-transfer squared at the proton vertex, $\Delta y$ the rapidity-gap width, and $y_{0}$ the center of the rapidity gap. In Eq. (6), the subscript $i=1,2$ enumerates Pomerons in a DPE event, $\Delta y=\Delta y_{1}+\Delta y_{2}$ is the total rapidity gap (sum of two gaps) in the event, and $y_{c}$ is the center in $\eta$ of the centrally-produced hadronic system.

The total cross section $\left(\sigma_{\text {tot }}\right)$ is expressed as:

$$
\begin{array}{lcl}
\sigma_{\text {tot }}^{p^{ \pm} p}= & 16.79 s^{0.104}+60.81 s^{-0.32} \mp 31.68 s^{-0.54} & \text { for } \sqrt{s} \leq 1.8 \mathrm{TeV}, \\
\sigma_{\text {tot }}^{p^{ \pm} p}= & \sigma_{\text {tot }}^{\mathrm{CDF}}+\frac{\pi}{s_{0}}\left[\left(\ln \frac{s}{s_{F}}\right)^{2}-\left(\ln \frac{s^{\mathrm{CDF}}}{s_{F}}\right)^{2}\right] & \text { for } \sqrt{s} \geq 1.8 \mathrm{TeV},
\end{array}
$$

where $s_{0}$ and $s_{F}$ are energy and the Pomeron flux saturation scales, respectively [7]. For $\sqrt{s} \leq 1.8$ $\mathrm{TeV}$, where there are Reggeon contributions, we use the global fit expression [9], while for $\sqrt{s} \geq 1.8$ $\mathrm{TeV}$, where Reggeon contributions are negligible, we employ the Froissart-Martin formula [10-12]. The two expressions are smoothly matched at $\sqrt{s} \sim 1.8 \mathrm{TeV}$.

The elastic cross section is obtained from the global fit [9] for $\sqrt{s} \leq 1.8 \mathrm{TeV}$, while for $1.8<$ $\sqrt{s} \leq 50 \mathrm{TeV}$ we use an extrapolation of the global-fit ratio of $\sigma_{\mathrm{el}} / \sigma_{\mathrm{tot}}$, which is slowly varying with $\sqrt{s}$, multiplied by $\sigma_{t o t}$. The total non-diffractive cross section is then calculated as $\sigma_{\mathrm{ND}}=$ $\left(\sigma_{\mathrm{tot}}-\sigma_{\mathrm{el}}\right)-\left(2 \sigma_{\mathrm{SD}}+\sigma_{\mathrm{DD}}+\sigma_{\mathrm{CD}}\right)$.

\section{Results}

In this section, we present as examples of the predictive power of the RENORM model some results reported by the TOTEM, CMS, and ALICE collaborations for $p p$ collisions at $\sqrt{s}=7 \mathrm{TeV}$, which can be directly compared with RENORM formulas without using the PYTHIA8-MBR simulation. Figure 1 (left) 
shows a comparison of the тотем total, elastic, and total-inelastic cross sections, along with results from other experiments fitted by the COMPETE Collaboration [13]; the RENORM predictions, displayed as filled (green) squares, are in excellent agreement with the тотем results. Similarly, in Fig. 1 (right), good agreement is observed between the ALICE [14] and CMS [15] inelastic cross sections and the RENORM prediction.

The uncertainty shown in the RENORM prediction of $\sigma_{\text {tot }}$ in Fig. 1 (left) is dominated by that in the scale parameter $s_{0}$. The latter can be reduced by a factor of $\sim 4$ if $\sqrt{s}_{0}$ is interpreted as the mean value of the glue-ball-like object discussed in [16] and the data shown in Fig. 8 of [16] are then used to determine its value. Work is in progress to finalize the details of this interpretation.
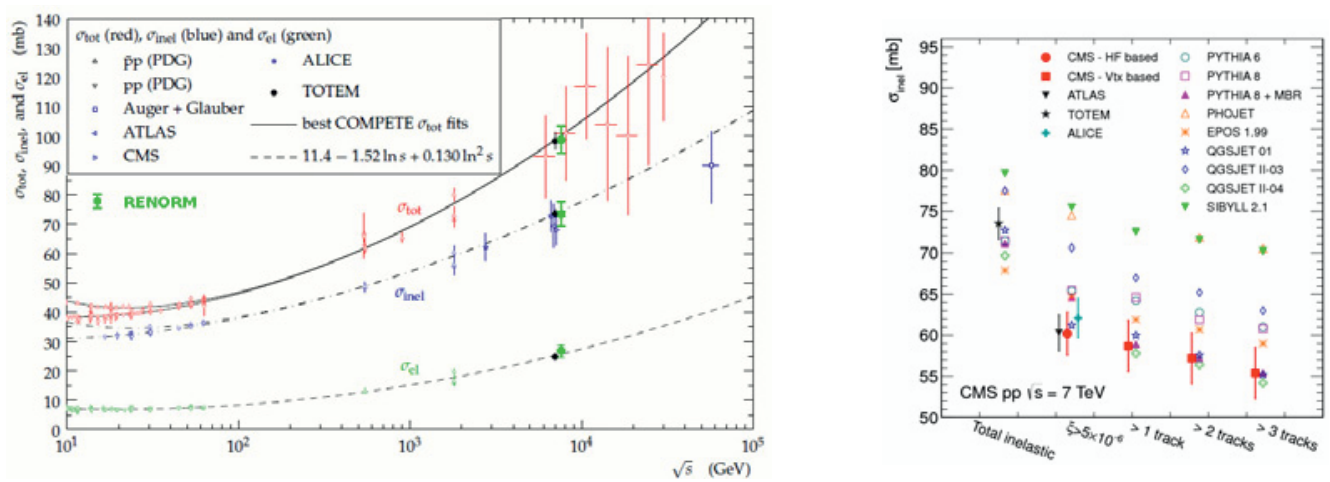

Figure 1. (left) тотем measurements of the total, total-inelastic, and elastic $p p$ cross sections at $\sqrt{s}=7 \mathrm{TeV}$ shown with best COMPETE fits [13] and RENORM predictions added as filled squares; (right) ALICE [14] and CMS [15] measurements of inelastic cross sections at $\sqrt{s}=7 \mathrm{TeV}$ are compared to RENORM predictions (PYTHIA8-MBR).
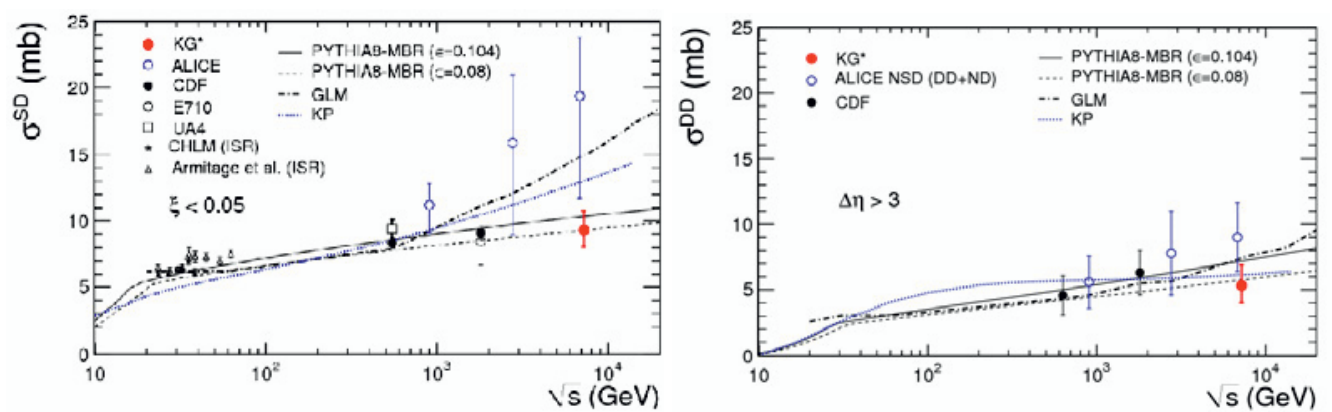

$\mathrm{KG}^{*}$ : this "data" point was obtained after extrapolation into the unmeasured low mass region(s) from the measured cMs cross sections [17] using the MBR model.

Figure 2. Measured SD (left) and DD (right) cross sections for $\xi<0.05$ compared with theoretical predictions; the model embedded in PYTHIA8-MBR provides a good description of all data.

Another example of the predictive power of RENORM is shown in Fig. 2, which displays the total SD (left) and total DD (right) cross sections vs the forward momentum loss $\xi$ of the diffracted proton $(\Delta y \approx-\ln \xi$ ) for $\xi<0.05$, after extrapolation into the low mass region from the measured cms cross sections at higher mass regions, presented in [17], using RENORM. 
The justification for using the RENORM model for the extrapolation into the low mass region is presented in Fig. 3, in which the measured diffractive croee sections within a wide (albeit limited) pseudorpidity regions are compared with predictions. In Figs. 3 (top-left and top-middle), the predictions of PYTHIA8-MBR are shown for two values of the $\epsilon$ parameter of the Pomeron trajectory $\left(\alpha(t)=1+\epsilon+\alpha^{\prime} t\right), \epsilon=0.08$ and $\epsilon=0.104$. Both values describe the measured SD cross section within uncertainties, while the DD data favor the smaller value of $\epsilon$, which is consistent with low mass CDF data. The predictions of PYTHIA8-4c and PYTHIA6 describe well the measured DD cross section, but fail to describe the falling behavior of the data (see details in [17]). The total SD cross cross section integrated over the region $-5.5<\log _{10} \xi<-2.5\left(12 \lesssim M_{X} \lesssim 394 \mathrm{GeV}\right)$ was measured to be $\sigma_{v i s}^{S D}=4.27 \pm 0.04$ (stat.) $)_{-0.58}^{+0.65}$ (syst.) $\mathrm{mb}$ (dissociation of either proton).

The event sample after the $\Delta \eta^{0}>3$ selection, was used to extract the differential DD cross section as a function of the central-gap width, $\Delta \eta$. The cross section for $\Delta \eta>3, \mathrm{M}_{X}>10 \mathrm{GeV}$ and $\mathrm{M}_{Y}>10$ $\mathrm{GeV}$ is presented in Fig. 3 (right). The total DD cross cross section integrated over this region was measured to be $\sigma_{v i s}^{D D}=0.93 \pm 0.01$ (stat.) ${ }_{-0.22}^{+0.26}$ (syst.) mb.
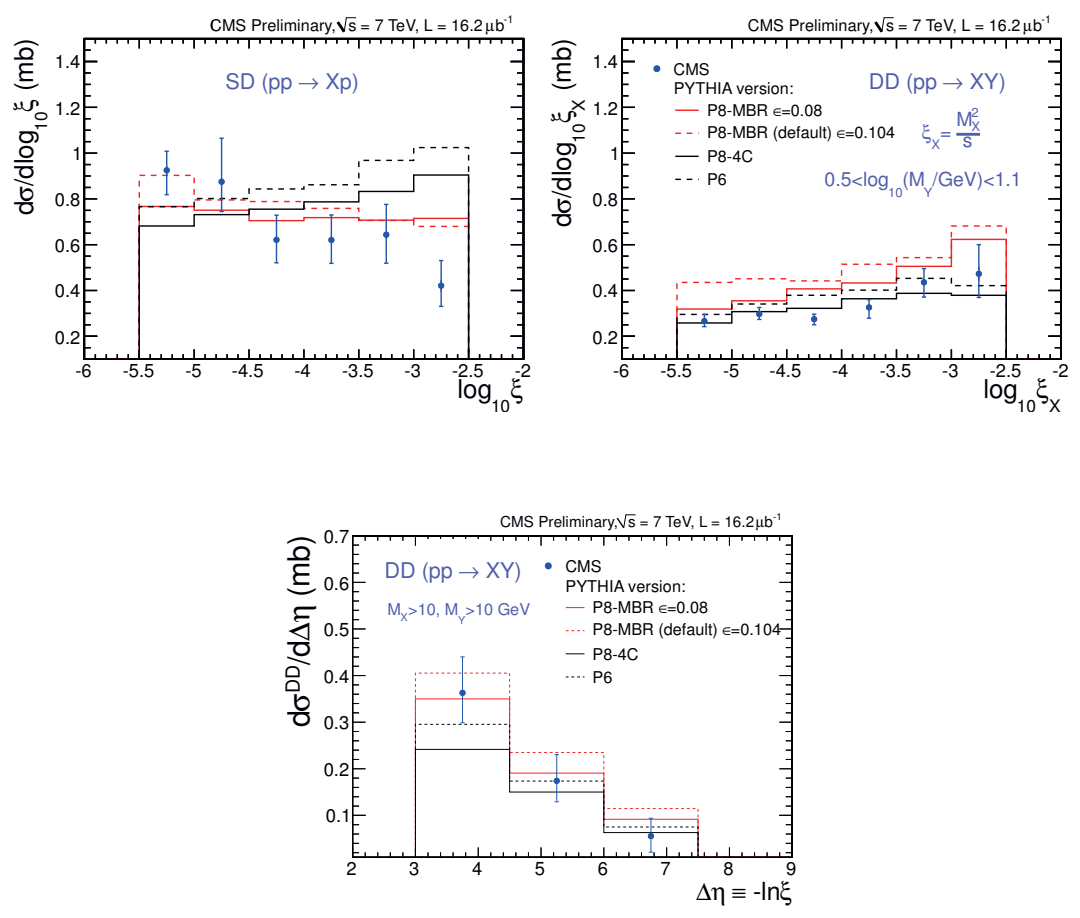

Figure 3. SD (top-left) and DD (top-right) cross sections vs $\xi$, and DD cross section vs $\Delta \eta$ (bottom), compared to PYTHIA6, PYTHIA8-4c and PYTHIA8-MBR MC. Errors are dominated by systematic uncertainties (HF calorimeter energy scale, and hadronization and diffraction model).

\section{Summary}

We reviewed our pre-LHC predictions for the total, elastic, total-inelastic, and diffractive components of the proton-proton cross section at high energies, which are based on a special parton-model approach to diffraction employing inclusive proton parton distribution functions and QCD color factors. 
We discuss single diffraction/dissociation, double diffraction/dissociation, and central diffraction or double-Pomeron exchange, comparing predictions with LHC measurements. Agreement between data and PYTHIA8-MBR predictions is found in all cases.

\section{Acknowledgments}

I would like to thank Robert Ciesielski, my colleaguea at Rockefeller and collaborator in the implementation of the MBR simulation into PYTHIA8, and the Office of Science of the Department of Energy for supporting the Rockefeller experimental diffraction physics programs at Fermilab and LHC on which this research is anchored.

\section{References}

[1] K. Goulianos, Predictions of Diffractive Cross Sections in Proton-Proton Collisions, in proceedings of Diffraction 2012: International Workshop on Diffraction in High Energy Physics, 10-15 September 2012, AIP Conf. Proc. 1523, 107 (2013) [doi:http://dx.doi.org/10.1063/1.4802128].

[2] See models presented by various authors in proceedings of Diffraction 2012, AIP Conf. Proc. 1523.

[3] K. Goulianos, Predictions of Diffractive, Elastic, Total, and Total-Inelastic pp Cross Sections vs LHC Measurements, to appear in proceedings of XXI International Workshop on Deep-Inelastic Scattering and Related Subject-DIS2013, 22-26 April 2013, Marseilles, France.

[4] K. Goulianos, Hadronic Diffraction: Where do we Stand?, in proceedings of Les Rencontres de Physique de la Vallee d'Aoste: Results and Perspectives in Particle Physics, La Thuile, Italy, February 27 - March 6, 2004, Frascati Physics Series, Special 34 Issue, edited by Mario Greco [arXiv:hep-ph/0407035].

[5] K. Goulianos, Diffractive and Total pp Cross Sections at LHC, in proceedings of 13th International Conference on Elastic and Diffractive Scattering (Blois Workshop) - Moving Forward into the LHC Era, CERN, Geneva, Switzerland, June 29-July 3, 2009, CERN-Proceedings-2010-02, edited by Mario Deile [arXiv:1002.3527v2].

[6] T. Sjöstrand, S. Mrenna and P. Skands, JHEP05 (2006) 026, Comput. Phys. Comm. 178 (2008) 852 [arXiv:hep-ph/0603175], [arXiv:0710.3820].

[7] R. Ciesielski and K. Goulianos, MBR Monte Carlo Simulation in PYTHIA8 [arXiv:1205.1446].

[8] K. Goulianos, Phys. Lett. B 193, 151 (1987).

[9] R. J. M. Covolan, J. Montanha and K. Goulianos, Phys. Lett. B 389, 176 (1996).

[10] M. Froissart, Phys. Rev. 3, 123 (1961).

[11] A. Martin, Nuovo Cimento 42, 930 (1966).

[12] A. Martin, Phys. Rev. D 80, 065013 (2009).

[13] M. Deile (тотем Collaboration), Measurements of Proton-Proton and Total Cross Section at the LHC by TOTEM, in proceedings of DIFFRACTION 2012 (figure from talk presented at that Conference).

[14] B. Abelev et al. (ALICE Collaboration), Measurement of Inelastic, Single- and Double-Diffraction Cross Sections in Proton-Proton Collisions at the LHC with ALICE, Eur. Phys. J. C (2013)73:2456, [arXiv:1208.4968], [DOI 10.1140/cpjc/s10052-013-2456-0]

[15] S. Chatrchyan et al. (CMS Collaboration), Measurement of the inelastic proto-proton cross section at $\sqrt{s}=7 \mathrm{TeV}$, Phys. Lett. B 722, 5 (2013). 


\section{EPJ Web of Conferences}

[16] M. G. Albrow, T. D. Coughlin, J. R. Forshaw, Central Exclusive Particle Production at High Energy Hadron Colliders, Prog. Part. Nucl. Phys 65, 149-184, 2010 [DOI: 10.1016/j.ppnp.2010.06.001].

[17] R. Ciesielski (cms Collaboration), Measurements of diffraction in p-p collisions in CMS, to appear in proceedings of XXI International Workshop on Deep-Inelastic Scattering and Related Subject-DIS2013, 22-26 April 2013, Marseilles,France. 